\title{
Sample Preparation for Powder Diffraction
}

The single most important step in powder diffraction analysis is the preparation of the sample. Unless the sample is representative of the material under study and meets all the other criteria to produce its representative diffraction pattern, the experimental data may be insufficient to provide the answers needed. Considerably more time may be spent trying to interpret the bad data than would have been necessary to prepare the proper sample in the first place.

The subject of sample preparation usually receives minimal attention in modern diffraction papers. This lack of discussion is more true in recent years now that we rely on the computer to assist in all steps from data collection to data analysis. Unfortunately, the computer cannot compensate for most bad data, and the initial sample is more important now than it has ever has been. Even the principal textbooks devote very little space to the topic of the preparation of samples for the many types of experiments that are discussed. (Note my comments in the book review of The Rietveld Method in the International Report section of this issue.) Consequently, Victor Burkhe, Ron Jenkins, and $I$ are assembling material for a book devoted entirely to the subject of sample preparation in X-ray analysis, both diffraction and fluorescence.

This book is partly a product of the workshops on sample preparation at the Denver X-ray Conferences and partly a long-term pet project of the author/editors involved. The goal of the book is to concentrate on the sample, how it is obtained from the bulk material under study, how it is comminuted and packed for presentation to the diffraction apparatus, and what properties it must have to yield good diffraction data. It will also cover types of samples other than powders, and samples which have special difficulties, such as limited quantities, atmospheric sensitivity, hazardous materials, etc.; and samples that are needed for unique experiments, such as clay minerals, forensic samples, radioactive materials, etc. This goal is very large, and we need your help.

The editors have already contacted many potential contributors with requests to provide appropriate descriptions for inclusion in the book. This editorial is to solicit additional material from the readers of POWDER DIF$F R A C T I O N$ who may not already have been contacted. To date the list of contributors who have indicated interest in participating in this project is close to 50 . There are 1000 readers of POWDER DIFFRACTION. In order to cover this extensive topic as fully as possible, we need all the help we can get. We also need YOU.

The format of the book is to be as simple and direct as possible in the presentation of sample preparation procedures. Most of the descriptions will be in recipe form like a cook book. They should list step-by-step each part of the procedure with annotations as to the equipment needed, sources of the equipment, and footnotes on each part explaining the purpose and goal of the step toward achieving the final sample. In addition to recipes, there will be discussions of samples designed for special purposes such as reference patterns for the Powder Diffraction File and the proper use of internal and external standards for calibration. The outline of potential topics for the diffraction section alone is over three pages already. Unfortunately, there are not authors for every topic in the list. Thus we are soliciting your assistance.

What we need are descriptions of procedures which you use in your laboratory. Many of these procedures are probably already written to meet company requirements. Other procedures are mentioned casually in notebooks. Still other procedures are in heads of the experimentalist. Regardless of the source or the simplicity of the procedure, we need this information. Do not consider the procedure too simple for inclusion in this book. We would rather receive duplications than omissions because the author considered the procedure too simple for submission. The editors will blend all the contributions into a coherent presentation and acknowledge the contributors appropriately.

Because readers of POWDER DIFFRACTION are often responsible for XRF analyses also, we encourage you to supply procedures for XRF as well. Submission is simple; just send your informal or formal writeup to one of the book editors. We also request that you supply reprints of any articles that contain information on sample preparation procedures. If you do not have reprints available or if you have some pet articles that you follow, please supply the reference. We can dig out the article easily if we know where to look. Do not put off this request. We need the information now.

The first deadline for information is essentially the day you receive this issue of POWDER DIFFRACTION. We would appreciate either the information immediately after you read this editorial or a letter with a short outline of the material you will supply. We plan to prepare the first draft of the text early in 1994 and to circulate it to all contributors for their input. We hope to have the final manuscript in the publishers' hands by mid 1994 for publication in late 1994 or early 1995 . This schedule is ambitious, but with your help we can achieve the goal as planned. Please respond immediately.

Deane K. Smith Editor-in-Chief 